\title{
A few remarks on the values of the Bernoulli polynomials at rational arguments and some relations with $\zeta(2 k+1)$
}

\author{
André Pierro de Camargo ${ }^{1}$ \\ and Giulliano Cogui de Oliveira Teruya ${ }^{2}$ \\ ${ }^{1}$ Federal University of the ABC region, Brazil \\ e-mail: andrecamargo.math@gmail.com \\ ${ }^{2}$ Federal University of the ABC region, Brazil \\ e-mail: giulliano.teruyadaluno.ufabc.edu.br
}

\begin{abstract}
A problem posed by Lehmer in 1938 asks for simple closed formulae for the values of the even Bernoulli polynomials at rational arguments in terms of the Bernoulli numbers. We discuss this problem based on the Fourier expansion of the Bernoulli polynomials. We also give some necessary and sufficient conditions for $\zeta(2 k+1)$ be a rational multiple of $\pi^{2 k+1}$.
\end{abstract}

Keywords: Bernoulli polynomials, Bernoulli numbers, Riemann zeta function, Euler's formula. 2020 Mathematics Subject Classification: 11B68, 11 M99.

\section{Introduction}

The Bernoulli numbers $B_{0}, B_{1}, B_{2}, \ldots$ is a sequence of rational numbers that has important applications in Analysis and Number theory. For instance, they appear in the Taylor expansion of the tangent and other related functions $[4,5]$ and in the Euler-Maclaurin summation formula [2]. The Bernoulli numbers are also the constant terms of the Bernoulli polynomials (see [7-10] for some recent research on Bernoulli polynomials). Among the various possible ways of defining the Bernoulli polynomials and numbers, one is [2]: 


$$
B_{0}=B_{0}(x):=1 \text { and } B_{m}(x)=\int_{0}^{x} m B_{m-1}(x)+B_{m}, m \geq 1,
$$

where $B_{m}$ is such that

$$
\int_{0}^{1} B_{m}(x) d x=0, m \geq 1 .
$$

For $n=1,2,3,4$ and 6 and $\ell \leq n$, the values of the Bernoulli polynomials $B_{k}\left(\frac{\ell}{n}\right), k$ even, are given by

$$
B_{k}(0)=B_{k}(1)=B_{k}, \quad B_{k}\left(\frac{1}{2}\right)=\left(2^{1-k}-1\right) B_{k}
$$

and

$$
\begin{aligned}
& B_{k}\left(\frac{1}{3}\right)=B_{k}\left(\frac{2}{3}\right)=\frac{1}{2}\left(3^{1-k}-1\right) B_{k}, \\
& B_{k}\left(\frac{1}{4}\right)=B_{k}\left(\frac{3}{4}\right)=\frac{1}{2}\left(4^{1-k}-2^{1-k}\right) B_{k}, \\
& B_{k}\left(\frac{1}{6}\right)=B_{k}\left(\frac{5}{6}\right)=\frac{1}{2}\left(6^{1-k}-3^{1-k}-2^{1-k}+1\right) B_{k} .
\end{aligned}
$$

The problem of determining whether $B_{k}\left(\frac{\ell}{n}\right), k$ even, has a simple closed form (like those above) for other irreducible fractions $\frac{\ell}{n}$ with $\ell<n$ is instigated by Lehmer in her paper of 1938 (see footnote in p. 352 of [12]) and also by Granville and Sun in the more recent paper [6] of 1996. Nevertheless, no significant advance with respect to this question seems to have been reported so far.

The cases contemplated in (3) are derived in [6] using the symmetries of $B_{2 k}(x)$ with respect to $x=\frac{1}{2}$ and Raabe's multiplication theorem, [1] p. 804,

$$
B_{k}(n x)=n^{k-1} \sum_{j=0}^{n-1} B_{k}\left(x+\frac{j}{n}\right), n \geq 1 .
$$

In the first enigmatic case, $n=5,(4)$ and the symmetries of $B_{2 k}(x)$ yield

$$
\left\{\begin{array}{rllc}
B_{2 k}\left(\frac{1}{5}\right)-B_{2 k}\left(\frac{4}{5}\right) & = & 0 \\
B_{2 k}\left(\frac{2}{5}\right)-B_{2 k}\left(\frac{3}{5}\right) & = & 0 \\
B_{2 k}\left(\frac{1}{5}\right)+B_{2 k}\left(\frac{2}{5}\right)+B_{2 k}\left(\frac{3}{5}\right)+B_{2 k}\left(\frac{4}{5}\right) & = & \left(\frac{1}{5^{2 k-1}}-1\right) B_{2 k},
\end{array}\right.
$$

so one may be tempting to launching in the quest of just another algebraic equation involving

$$
B_{2 k}\left(\frac{1}{5}\right), B_{2 k}\left(\frac{2}{5}\right), B_{2 k}\left(\frac{3}{5}\right) \text { and } B_{2 k}\left(\frac{4}{5}\right)
$$

in order to solve the question.

In this note we call the attention to the impossibility of expressing the ratio $\frac{B_{2 k}\left(\frac{\ell}{n}\right)}{B_{2 k}}$ as a rational function of powers of $k$ with rational coefficients, like the expressions in (3). In fact,

Lemma 1.1. For $x \in[0,1]$, we have

$$
\lim _{k \rightarrow \infty} \frac{B_{2 k}(x)}{B_{2 k}}=\cos (2 \pi x)
$$


In particular, for $x=\frac{1}{5},(7)$ yields

$$
\lim _{k \rightarrow \infty} \frac{B_{2 k}\left(\frac{1}{5}\right)}{B_{2 k}}=\frac{-1+\sqrt{5}}{4} .
$$

Since $\frac{B_{2 k}\left(\frac{1}{5}\right)}{B_{2 k}}$ is always a rational number, this shows that it is impossible to express this ratio as a rational function of powers of $k$ with rational coefficients, like the expressions in (3).

Equation (7) follows directly by the Fourier representation of the Bernoulli polynomials (see a proof below in the fold). A more informative expression for $B_{2 k}\left(\frac{\ell}{n}\right)$ can be obtained in the same manner in terms of the modular fractions of the Riemann zeta function:

$$
\mu_{\zeta}(z, n, q):=\frac{\sum_{j=0}^{\infty} \frac{1}{[n j+q]^{z}}}{\sum_{j=1}^{\infty} \frac{1}{j^{z}}}=\frac{\sum_{j=0}^{\infty} \frac{1}{[n j+q]^{z}}}{\zeta(z)}, q=1,2, \ldots, n, \mathfrak{R} e(z)>1
$$

For $k \geq 1, n \geq 2, \ell=1,2, \ldots, n$, and for $\mu_{\zeta}$ defined in (9),

$$
B_{2 k}\left(\frac{\ell}{n}\right)=B_{2 k}\left(\sum_{q=1}^{n} \mu_{\zeta}(2 k, n, q) \cos \left(\frac{2 q \ell \pi}{n}\right)\right) .
$$

We have independently come across (10), but this result is not new ${ }^{1}$. A slightly different version of (10) can be found in [3] in terms of the Hurwitz zeta function. We note that (10) can be used to give an analytic proof of (3):

Corollary 1.1.1. The values $B_{k}\left(\frac{1}{2}\right), B_{k}\left(\frac{1}{3}\right), B_{k}\left(\frac{2}{3}\right), B_{k}\left(\frac{1}{4}\right), B_{k}\left(\frac{3}{4}\right), B_{k}\left(\frac{1}{6}\right)$ and $B_{k}\left(\frac{5}{6}\right)$ are given by the right-hand side of (3).

Therefore, it seems that (10) gives possibly the simplest closed form for the values $B_{2 k}\left(\frac{\ell}{n}\right)$ in the sense that the right-hand side of (10) is a linear combination of a finite number of functions of $k$ that are somewhat familiar.

A better known problem than the one discussed above is about the irrationality of the values of the Riemann zeta function at odd positive integers. Until now, only $\zeta(3)$ has been known to be irrational. A closely related open question, inspired by Euler's formula for $\zeta(2 k)$, is determining whether the numbers $\beta_{2 k+1}$ defined by

$$
\zeta(2 k+1)=\beta_{2 k+1}(-1)^{k} \frac{2^{2 k} \pi^{2 k+1}}{(2 k+1) !}, k \geq 1
$$

are rational or not [5]. We show that some of the modular fractions $\mu_{\zeta}(2 k+1, n, q)$ defined by (9) are closely related to the numbers $\beta_{2 k+1}$ defined by (11). More precisely, we have

Theorem 1.2. For $k \geq 1$,

\footnotetext{
${ }^{1}$ It is also worth noting that the maxima and minima of Bernoulli polynomials were previously analyzed using Fourier expansions [11].
} 


$$
\begin{gathered}
\mu_{\zeta}(2 k+1,3,1)=\frac{1}{2}\left(1-3^{-2 k-1}\right)+\frac{1}{\sqrt{3}} \frac{B_{2 k+1}\left(\frac{1}{3}\right)}{\beta_{2 k+1}}, \\
\mu_{\zeta}(2 k+1,3,2)=\frac{1}{2}\left(1-3^{-2 k-1}\right)-\frac{1}{\sqrt{3}} \frac{B_{2 k+1}\left(\frac{1}{3}\right)}{\beta_{2 k+1}}, \\
\mu_{\zeta}(2 k+1,4,1)=\frac{1}{2}-2^{-2 k-2}+\frac{1}{2} \frac{B_{2 k+1}\left(\frac{1}{4}\right)}{\beta_{2 k+1}}, \\
\mu_{\zeta}(2 k+1,4,3)=\frac{1}{2}-2^{-2 k-2}-\frac{1}{2} \frac{B_{2 k+1}\left(\frac{1}{4}\right)}{\beta_{2 k+1}}, \\
\mu_{\zeta}(2 k+1,6,1)=\frac{1}{2}\left[1-\frac{1}{2^{2 k+1}}-\frac{1}{3^{2 k+1}}+\frac{1}{6^{2 k+1}}\right]+\frac{1}{\sqrt{3}}\left[\frac{B_{2 k+1}\left(\frac{1}{6}\right)}{\beta_{2 k+1}}-\frac{1}{2^{2 k+1}} \frac{B_{2 k+1}\left(\frac{1}{3}\right)}{\beta_{2 k+1}}\right], \\
\mu_{\zeta}(2 k+1,6,5)=\frac{1}{2}\left[1-\frac{1}{2^{2 k+1}}-\frac{1}{3^{2 k+1}}+\frac{1}{6^{2 k+1}}\right]-\frac{1}{\sqrt{3}}\left[\frac{B_{2 k+1}\left(\frac{1}{6}\right)}{\beta_{2 k+1}}-\frac{1}{2^{2 k+1}} \frac{B_{2 k+1}\left(\frac{1}{3}\right)}{\beta_{2 k+1}}\right] .
\end{gathered}
$$

Theorem 1.2 shows that $\beta_{2 k+1}$ is rational if and only if $\mu_{\zeta}(2 k+1,4,1)$ or $\mu_{\zeta}(2 k+1,4,3)$ are rationals. In the affirmative case, it also shows that $\mu_{\zeta}(2 k+1, n, q)$ sometimes is rational, sometimes is not.

In the rest of the note, we prove Theorem 1.2, Lemma 1.1 and Corollary 1.1.1.

\section{Proofs}

\subsection{Proof of Theorem 1.2}

Our proof is based on the Fourier expansion of the Bernoulli polynomials, [1] p. 805:

$$
\left.B_{2 k-1}(x)=(-1)^{k} \frac{2(2 k-1) !}{(2 \pi)^{2 k-1}} \sum_{j=1}^{\infty} \frac{\sin (2 j \pi x)}{j^{2 k-1}}, x \in\right] 0,1[\text {, for } k=1,
$$

$x \in[0,1]$, for $k>1$, and

$$
B_{2 k}(x)=(-1)^{k+1} \frac{2(2 k) !}{(2 \pi)^{2 k}} \sum_{j=1}^{\infty} \frac{\cos (2 j \pi x)}{j^{2 k}}, x \in[0,1], \quad k \geq 1 .
$$

Let $x_{\ell}=\frac{\ell}{n}, \ell=0,1,2, \ldots, n$. We have

$$
\begin{aligned}
\frac{B_{2 k+1}\left(x_{\ell}\right)}{\beta_{2 k+1}} \stackrel{(11),(12)}{=} \frac{1}{\zeta(2 k+1)} \sum_{j=1}^{\infty} \frac{\sin \left(2 j \pi x_{\ell}\right)}{j^{2 k+1}} \\
=\frac{1}{\zeta(2 k+1)} \sum_{q=1}^{n} \sum_{j=0}^{\infty} \frac{\sin \left(2[n j+q] \pi x_{\ell}\right)}{[n j+q]^{2 k+1}} \\
=\sum_{q=1}^{n} \mu_{\zeta}(2 k+1, n, q) \sin \left(\frac{2 q \ell \pi}{n}\right) .
\end{aligned}
$$

To simplify the notation, we shall write only $\mu_{\zeta}(n, q)$ instead of $\mu_{\zeta}(2 k+1, n, q)$. First, note that

$$
\mu_{\zeta}(n, n)=\frac{1}{n^{2 k+1}} \text { and } \mu_{\zeta}(a n, a q)=\frac{1}{a^{2 k+1}} \mu_{\zeta}(n, q)
$$


For $n=3$, (14) gives

$$
\begin{aligned}
\frac{B_{2 k+1}\left(\frac{1}{3}\right)}{\beta_{2 k+1}} & =\sin \left(\frac{2 \pi}{3}\right) \mu_{\zeta}(3,1)+\sin \left(\frac{4 \pi}{3}\right) \mu_{\zeta}(3,2)+\sin \left(\frac{6 \pi}{3}\right) \mu_{\zeta}(3,3), \\
& =\frac{\sqrt{3}}{2} \mu_{\zeta}(3,1)-\frac{\sqrt{3}}{2} \mu_{\zeta}(3,2)
\end{aligned}
$$

In addition,

$$
\begin{array}{r}
1 \stackrel{=}{=} \mu_{\zeta}(3,1)+\mu_{\zeta}(3,2)+\mu_{\zeta}(3,3) \\
\stackrel{(15)}{=} \mu_{\zeta}(3,1)+\mu_{\zeta}(3,2)+3^{-2 k-1} .
\end{array}
$$

Therefore,

$$
\left\{\begin{array}{l}
\mu_{\zeta}(3,1)=\frac{1}{2}\left(1-3^{-2 k-1}\right)+\frac{1}{\sqrt{3}} \frac{B_{2 k+1}\left(\frac{1}{3}\right)}{\beta_{2 k+1}} \\
\mu_{\zeta}(3,2)=\frac{1}{2}\left(1-3^{-2 k-1}\right)-\frac{1}{\sqrt{3}} \frac{B_{2 k+1}\left(\frac{1}{3}\right)}{\beta_{2 k+1}}
\end{array}\right.
$$

For $n=4$, (14) gives

$$
\begin{aligned}
\frac{B_{2 k+1}\left(\frac{1}{4}\right)}{\beta_{2 k+1}} & =\sin \left(\frac{2 \pi}{4}\right) \mu_{\zeta}(4,1)+\sin \left(\frac{4 \pi}{4}\right) \mu_{\zeta}(4,2)+\sin \left(\frac{6 \pi}{4}\right) \mu_{\zeta}(4,3)+\sin \left(\frac{8 \pi}{4}\right) \mu_{\zeta}(4,4) \\
& =\mu_{\zeta}(4,1)-\mu_{\zeta}(4,3)
\end{aligned}
$$

In addition,

$$
\begin{aligned}
1 & =\mu_{\zeta}(4,1)+\mu_{\zeta}(4,2)+\mu_{\zeta}(4,3)+\mu_{\zeta}(4,4) \\
& \stackrel{(15)}{=} \mu_{\zeta}(4,1)+\mu_{\zeta}(4,3)+\frac{1}{2^{2 k+1}} \mu_{\zeta}(2,1)+\frac{1}{4^{2 k+1}} \\
& \stackrel{(15)}{=} \mu_{\zeta}(4,1)+\mu_{\zeta}(4,3)+\frac{1}{2^{2 k+1}}\left[1-\frac{1}{2^{2 k+1}}\right]+\frac{1}{4^{2 k+1}} .
\end{aligned}
$$

Therefore,

$$
\left\{\begin{array}{l}
\mu_{\zeta}(4,1)=\frac{1}{2}-2^{-2 k-2}+\frac{1}{2} \frac{B_{2 k+1}\left(\frac{1}{4}\right)}{\beta_{2 k+1}} \\
\mu_{\zeta}(4,3)=\frac{1}{2}-2^{-2 k-2}-\frac{1}{2} \frac{B_{2 k+1}\left(\frac{1}{4}\right)}{\beta_{2 k+1}}
\end{array}\right.
$$

For $n=6,(14)$ gives

$$
\begin{aligned}
\frac{B_{2 k+1}\left(\frac{1}{6}\right)}{\beta_{2 k+1}}= & \sin \left(\frac{2 \pi}{6}\right) \mu_{\zeta}(6,1)+\sin \left(\frac{4 \pi}{6}\right) \mu_{\zeta}(6,2)+\sin \left(\frac{6 \pi}{6}\right) \mu_{\zeta}(6,3) \\
& +\sin \left(\frac{8 \pi}{6}\right) \mu_{\zeta}(6,4)+\sin \left(\frac{10 \pi}{6}\right) \mu_{\zeta}(5,6)+\sin \left(\frac{12 \pi}{6}\right) \mu_{\zeta}(6,6) \\
= & \frac{\sqrt{3}}{2} \mu_{\zeta}(6,1)+\frac{\sqrt{3}}{2} \mu_{\zeta}(6,2)-\frac{\sqrt{3}}{2} \mu_{\zeta}(6,4)-\frac{\sqrt{3}}{2} \mu_{\zeta}(6,5) \\
& \stackrel{(15)}{=} \frac{\sqrt{3}}{2} \mu_{\zeta}(6,1)+\frac{\sqrt{3}}{2} \frac{1}{2^{2 k+1}} \mu_{\zeta}(3,1)-\frac{\sqrt{3}}{2} \frac{1}{2^{2 k+1}} \mu_{\zeta}(3,2)-\frac{\sqrt{3}}{2} \mu_{\zeta}(6,5) \\
& \stackrel{(16)}{=} \frac{\sqrt{3}}{2} \mu_{\zeta}(6,1)-\frac{\sqrt{3}}{2} \mu_{\zeta}(6,5)+\frac{1}{2^{2 k+1}} \frac{B_{2 k+1}\left(\frac{1}{3}\right)}{\beta_{2 k+1}} .
\end{aligned}
$$


In addition,

$$
\begin{aligned}
& 1=\mu_{\zeta}(6,1)+\mu_{\zeta}(6,2)+\mu_{\zeta}(6,3)+\mu_{\zeta}(6,4)+\mu_{\zeta}(6,5)+\mu_{\zeta}(6,6) \\
& \stackrel{(15)}{=} \mu_{\zeta}(6,1)+\frac{1}{2^{2 k+1}} \mu_{\zeta}(3,1)+\frac{1}{3^{2 k+1}} \mu_{\zeta}(2,1)+\mu_{\zeta} \frac{1}{2^{2 k+1}}(3,2) \\
& +\mu_{\zeta}(6,5)+\mu_{\zeta}(6,6) \\
& =\mu_{\zeta}(6,1)+\frac{1}{3^{2 k+1}}\left[1-\mu_{\zeta}(2,2)\right]+\frac{1}{2^{2 k+1}}\left[1-\mu_{\zeta}(3,3)\right] \\
& +\mu_{\zeta}(6,5)+\mu_{\zeta}(6,6) \\
& \stackrel{(15)}{=} \mu_{\zeta}(6,1)+\mu_{\zeta}(6,5)+\frac{1}{3^{2 k+1}}\left[1-\frac{1}{2^{2 k+1}}\right]+\frac{1}{2^{2 k+1}}\left[1-\frac{1}{3^{2 k+1}}\right]+\frac{1}{6^{2 k+1}} .
\end{aligned}
$$

Therefore,

$$
\left\{\begin{array}{l}
\mu_{\zeta}(6,1)=\frac{1}{2}\left[1-\frac{1}{2^{2 k+1}}-\frac{1}{3^{2 k+1}}+\frac{1}{6^{2 k+1}}\right]+\frac{1}{\sqrt{3}}\left[\frac{B_{2 k+1}\left(\frac{1}{6}\right)}{\beta_{2 k+1}}-\frac{1}{2^{2 k+1}} \frac{B_{2 k+1}\left(\frac{1}{3}\right)}{\beta_{2 k+1}}\right] \\
\mu_{\zeta}(6,5)=\frac{1}{2}\left[1-\frac{1}{2^{2 k+1}}-\frac{1}{3^{2 k+1}}+\frac{1}{6^{2 k+1}}\right]-\frac{1}{\sqrt{3}}\left[\frac{B_{2 k+1}\left(\frac{1}{6}\right)}{\beta_{2 k+1}}-\frac{1}{2^{2 k+1}} \frac{B_{2 k+1}\left(\frac{1}{3}\right)}{\beta_{2 k+1}}\right] .
\end{array}\right.
$$

\subsection{Proof of Corrolary 1.1.1}

Let us prove (3) for $\frac{\ell}{n}=\frac{1}{3}$ (the other cases are analogous). By (10), we have

$$
\frac{B_{2 k}\left(\frac{1}{3}\right)}{B_{2 k}}=\frac{F-\frac{1}{2} E}{E+F},
$$

with

$$
E=\sum_{j=0}^{\infty} \frac{1}{(3 j+1)^{2 k}}+\sum_{j=0}^{\infty} \frac{1}{(3 j+2)^{2 k}}, \quad F=\sum_{j=1}^{\infty} \frac{1}{(3 j)^{2 k}}
$$

However,

$$
F=\frac{1}{3^{2 k}} \sum_{j=1}^{\infty} \frac{1}{(j)^{2 k}}=\frac{1}{3^{2 k}}(E+F)
$$

Therefore

$$
\frac{B_{2 k}\left(\frac{1}{3}\right)}{B_{2 k}}=\frac{F\left(1+\frac{1}{2}\left[1-3^{2 k}\right]\right)}{3^{2 k} F}=\frac{1}{2}\left(3^{1-2 k}-1\right) .
$$

\subsection{Proof of Lemma 1.1}

By (13), for $x \in[0,1]$,

$$
\frac{B_{2 k}(x)}{B_{2 k}}=\frac{B_{2 k}(x)}{B_{2 k}(0)}=\frac{1}{\zeta(2 k)} \sum_{j=1}^{\infty} \frac{\cos (2 j \pi x)}{j^{2 k}}
$$

and we also have $\lim _{k \rightarrow \infty} \zeta(2 k)=1$ and $\lim _{k \rightarrow \infty} \sum_{j=1}^{\infty} \frac{\cos (2 j \pi x)}{j^{2 k}}=\cos (2 \pi x)$. 


\section{Summary}

In this note we investigated the impossibility of expressing the values of the Bernoulli polynomials $B_{2 k}\left(\frac{\ell}{n}\right)$ at rational arguments as products of the Bernoulli numbers $B_{2 k}$ and rational functions of powers of $k$ with rational coefficients. Lemma 1.1 tells us that this can not be done in general. We also give some necessary and sufficient conditions for $\zeta(2 k+1)$ be a rational multiple of $\pi^{2 k+1}$ in terms of the modular fractions $\mu_{\zeta}(2 k+1, n, q)$ defined by (9).

\section{References}

[1] Abramowitz, M., \& Stegun, I. (1972). Handbook of Mathematical Functions with Formulas, Graphs and Mathematical Tables. US Government Printing Office, Washington.

[2] Apostol, T. M. (1999). An elementary view of Euler-Maclaurin summation formula. The American Mathematical Monthly, 106(5), 409-418.

[3] Cvijovic, D., \& Klinowski, J. (1995). New formulae for the Bernoulli and Euler polynomials at rational arguments. Proceedings of the American Mathematical Society, 123(5), 1527-1535.

[4] De Amo, E., Carillo, M. D., \& Fernández-Sánchez, J. (2011). Another proof of Euler's formula for $\zeta(2 k)$. Proceedings of the American Mathematical Society, 139(4), 1441-1444.

[5] Dwilewicz, R. J., \& Minác̃, J. (2009). Values of the Riemann zeta function at integers. MATerials MATemàtics, 2009, Article number: 6, 26 pages.

[6] Granville, A., \& Sun, Z. (1996). Values of Bernoulli polynomials. Pacific Journal of Mathematics, 172(1), 117-137.

[7] Kim, T. (2008). Euler numbers and polynomials associated with zeta functions, Abstract and Applied Analysis, 2008, Article number: 581582, 11 pages.

[8] Kim, T., Kim, D. S., Dolgy, D. V., Lee, S. H., \& Kwon, J. (2021). Some Identities of the Higher-Order Type 2 Bernoulli Numbers and Polynomials of the Second Kind. CMES Computer Modeling in Engineering \& Sciences, 128(3), 1121-1132.

[9] Kim, T., Kim, D. S., Jang L. C., Lee, H., \& Kim, H. (2021). Generalized degenerate Bernoulli numbers and polynomials arising from Gauss hypergeometric function. Advances in Difference Equations, Article number: 175 (2021), 12 pages.

[10] Kim, T., Kim, D. S., Kwon., \& Lee, H. (2021). Representations of degenerate poly Bernoulli polynomials. Journal of Inequalities and Applications, Article number: 58 (2021), 12 pages.

[11] Lehmer, D. H. (1940). On the maxima and minima of Bernoulli polynomials. The American Mathematical Monthly, 47(8), 533-538.

[12] Lehmer, E. (1938). On congruences involving Bernoulli numbers and the quotients of Fermat and Wilson. Annals of Mathematics. Second Series, 39(2), 350-360. 